\title{
Tangence
}

\section{Dans les yeux d'un duc et pair. De l'autoportrait mélancolique à la genèse des Maximes}

\section{Éric Van der Schueren}

Numéro 60, mai 1999

L'éloquence du corps sous l'Ancien Régime

URI : https://id.erudit.org/iderudit/008080ar

DOI : https://doi.org/10.7202/008080ar

Aller au sommaire du numéro

Éditeur(s)

Presses de l'Université du Québec

ISSN

0226-9554 (imprimé)

1710-0305 (numérique)

Découvrir la revue

Citer cet article

Van der Schueren, É. (1999). Dans les yeux d'un duc et pair. De l'autoportrait mélancolique à la genèse des Maximes. Tangence, (60), 37-63.

https://doi.org/10.7202/008080ar d'utilisation que vous pouvez consulter en ligne.

https://apropos.erudit.org/fr/usagers/politique-dutilisation/ 


\title{
Dans les yeux d'un duc et pair. De l'autoportrait mélancolique à la genèse des Maximes \\ Éric Van der Schueren, Université Laval
}

\author{
À Raymond Joly, \\ en signe d'amitié et de respect, et tout à la joie \\ de la redondance des mots \\ dont l'un suffit à faire entendre l'autre \\ dans une sincère évidence.
}

... et il y a longtemps que j'ai éprouvé que la philosophie ne fait des merveilles que contre les maux passés ou contre ceux qui ne sont pas prêts d'arriver, mais qu'elle n'a pas grande vertu contre les maux présents.

La Rochefoucauld, le 24 octobre [1660].

Deux lignes fractionnent le discours critique contemporain sur les Maximes de La Rochefoucauld. La première sépare, avec plus ou moins d'étanchéité, les analyses qui voient dans le recueil du moraliste un exercice purement mondain ${ }^{1}$, à l'opposé doctrinal des critiques qui lui assignent une place de choix dans la production janséniste du milieu du $\mathrm{XVII}^{\mathrm{e}}$ siècle $^{2}$. La seconde ligne passe là et ailleurs, et Jean Starobinski est sans doute le plus fin au double jeu dans sa lecture qui situe l'origine des Maximes dans l'expérience amère de l'échec politique et qui les lit dans l'effacement du lieu et du temps de leur origine ${ }^{3}$, alors que la critique se disperse entre les

1 À l'exemple de Louis van Delft, "Pour une lecture mondaine de La Rochefoucauld. La caractériologie d'un moraliste pair de France", dans Images de La Rochefoucauld. Actes du tricentenaire 1680-1980, Paris, Presses universitaires de France, 1984, p. 145-157.

2 Jean Mesnard, "La rencontre de La Rochefoucauld avec Port-Royal", ibid., p. 161-165.

3 Voir, dans l'ordre, l'introduction aux Maximes et Mémoires, Paris, Union générale d'éditions, 1964 et "La Rochefoucauld ou l'oubli des secrets", Médecine de France, 1959, n 107, p. 33-40. 
38

tenants d'une lecture positive qui a élargi la lecture primitive par clés et ceux qui, retenant l'effet généralisant de la maxime, en font le point de fuite d'une glose atemporelle où l'auteur du recueil et son contexte disparaissent. Trop antithétiques, les positions critiques - qu'elles soient fondées ou non, il n'importe ici - paraissent inconciliables ${ }^{4}$. Deux cultures s'affrontent, celle de l'augustinisme anti-mondain et celle de la sociabilité ludique des salons, avant que ne les recouvrent des positions critiques qui puisent à des épistémès hostiles. Et si La Rochefoucauld n'était ni un janséniste froid, ni un salonnard brillant, pour autant que la question ait un intérêt... Et si les Maximes ne visaient à propulser le discours augustinien en une forme plus acceptable par les dévots des plaisirs de la terre, pas plus qu'elles ne seraient que le résultat d'une mode du siècle... Et si La Rochefoucauld était bien là, à chacune de ses Maximes, mais sans les atours attendus, tel Alceste, mélancolique à en crever et chamarré de rubans inopinément verts.

Il n'est nullement question ici de se jouer à nouveau des allusions cryptées du Misanthrope à l'un de ses contemporains, indécidables en ce qu'elles pointeraient le moraliste ou le duc de Montausier $^{5}$. Au positivisme vieilli de l'histoire littéraire ${ }^{6}$, cette étude préfère, sans irrespect aucun, les voies intuitives de l'analogie et de la métaphore proliférantes, toutefois guidée par la référence stable à Aristote: pareille référence construit un réseau

4 Après la thèse de Jean Lafond (La Rochefoucauld, augustinisme et littérature, Paris, Klincksieck, coll. "Bibliothèque française et romane", 1977) qui restitue les Maximes comme le fait de la rencontre des deux traditions fondatrices chrétienne et païenne, au filtre du thomisme et de la scolastique —, de la culture classique, l'analyse de Philippe Sellier ("La Rochefoucauld et la préciosité", dans Claire Carlin (dir.), La Rochefoucauld, Mithridate, Frères et sœurs, Les muses sœurs. Actes du $29^{e}$ Congrès annuel de la North American Society for Seventeenth-Century French Literature [...], Tübingen, Gunter Narr Verlag, coll. "Biblio 17", vol. CXI, 1998, p. 13-19) est sans doute l'une des plus perspicaces et des plus justes dans la réponse qui est faite au paradoxe de la figure d'un moraliste augustinien au milieu des plaisirs mondains de la civilité, qui a généré l'ambivalence du discours critique, lorsqu'elle rapporte les Maximes aux leçons de la préciosité, éthique mondaine, certes, mais aussi éthique du vrai, du pur et du constant.

5 Je suis en cela la voie tracée avec intelligence par R. Grandsaignes d'Hauterive, Le pessimisme de La Rochefoucauld, Paris, Librairie Armand Colin, 1914.

6 Je n'entends pas ici englober le travail fondamental de Henry A. Grubbs, "La genèse des "Maximes" de La Rochefoucauld", Revue d'bistoire littéraire de la France, 1932 , t. $39, \mathrm{n}^{\circ} 4$, p. $481-499$ et 1933 , t. $40, \mathrm{n}^{\circ} 1$, p. 17-37. 
serré qui, s'il ne rend pas compte de toute la finalité des Maximes, les place au centre d'un écheveau où concourent éthique, rhétorique et théorie des passions dans une liaison que le Stagyrite n'a cessé de nouer ${ }^{7}$. Ainsi sont rapportés à une doxa commune la mélancolie du moraliste, comme source créatrice à travers les théories du Problème $X X X$, 1, le genre de la maxime comme forme dérivée de l'enthymème dialectique et la dénonciation de l'amour-propre dont l'Éthique à Nicomaque (IX, 4 et 9, en particulier) préfigure le portrait que le duc devait en faire dans la maxime qui ouvre l'édition première; et cette unité de savoirs déplace du même fait la problématique de l'œuvre du moraliste dans un tout autre espace de préventions que celui des oppositions qui étaient évoquées d'entrée de jeu.

Le scandale des Maximes, bien connu aujourd'hui, tient évidemment au fait d'avoir joué d'une forme qui, depuis Aristote et surtout Quintillien, visait à soutenir et célébrer un savoir commun, sinon clanique, pour faire entendre, sous la plume de La Rochefoucauld une série de paradoxes pour le moins désobligeants. L'édition clandestine des Mémoires en Hollande, qui fut suivie après 1662 de bien d'autres, avait provoqué l'indignation de ceux qui, ayant pris une part aux événements de la Fronde, y retrouvaient leur portrait et l'explicitation de certaines de leurs motivations. Maximes et Mémoires paraissent ainsi liées sous le même sceau du scandale. Mais les deux textes le paraissent davantage encore dans leur énonciation qui tend à oblitérer le je locuteur ${ }^{8}$. Pareil effacement se trouve en fait validé dans la

7 Si Richard Lockwood ("How to read a Maxim", dans Claire Carlin (dir.), op. cit., p. 31-42) piste l'apparition du genre des maximes à la source de la Rhétorique d'Aristote, il n'en saisit pas les implications éthiques, qui sont pourtant évidentes dans le texte du philosophe grec et où se produit la liaison de la sentence avec un contenu moral. Voir, entre autres, la conclusion sur la maxime - en forme d'enthymème, bien sûr - dans la Rhétorique (1395b): "Le caractère moral se relève dans les discours où l'intention de l'orateur se manifeste; or, toute sentence atteint ce résultat en faisant paraître l'orateur comme énonçant une sentence générale à propos de l'objet particulier de ses intentions: de sorte que, si les sentences sont honnêtes, elles donnent aux moeurs de l'orateur une apparence honnête aussi" (dans la traduction de C. É. Ruelle, revue par P. Vanhemelryck, Paris, Livre de Poche, 1991, p. 260). Et comme les sentences de La Rochefoucauld parurent, pour certaines, déshonnêtes, les lecteurs ne manquèrent pas de lui en attribuer tous les vices... J'y reviendrai.

8 On sait que La Rochefoucauld composa d'abord les livres III à VI de ses Mémoires, où, parlant de lui-même, il reprend l'énonciation césarienne de la 
40

manipulation réservée que le duc voulut pour ses œuvres: comme les Maximes, les Mémoires ont circulé en manuscrits dans le réseau étroit des amitiés sûres, complices et souvent féminines. De même encore, l'autoportrait que le duc se résolut à composer en 1658 prenait place dans un recueil que voulut la Grande Mademoiselle dans un tirage restreint et électif. Mais comme cela se produisit pour les Mémoires, il y eut des indiscrétions, des fuites et finalement des éditions plus ou moins correctes qui ont privé les textes des voies intimistes et prestigieuses de la lecture choisie entre pairs. Avant de souscrire à la mode du portrait littéraire et à celle de la maxime qui lui succédera, La Rochefoucauld avait répondu à la vogue des portraits en émail que Petitot, réfugié en France à la suite de la destitution de Charles Ier, suscita dans le Paris de la Fronde?. Mode féminine par prévention, le portrait en émail soustrait sa manipulation aux rites exhibitionnistes de la peinture sur toile et son petit format est gage d'intimité. Les rapports du duc au symbolique sont ainsi cohérents en ce qu'ils se placent toujours en référence de pratiques directement déduites d'un repli sur le privé au sein du cercle des amitiés proches.

La publication des Maximes, pleinement contrôlée cette fois, se comprend alors par effet inverse de la préférence intimiste: en plus de l'anonymat, leur énonciation abstraite protège le lieu privé des consciences qui ont conspiré à la rédaction du recueil, en premier lieu Madame de Sablé et les frères Esprit. En surcroît, forme brève du dilettantisme honnête ${ }^{10}$, la maxime permet à La Rochefoucauld de respecter pleinement les préventions de ce que Bouhours définira comme le bel esprit, mêlant prestige symbolique et aristocratisme social, et de n'empiéter point sur les compétences des

troisième personne, qu'il abandonnera dans les deux premiers livres. Sur l'effacement du locuteur dans les Maximes, voir la mise au point — aux fins de la rhétorique - de Béatrice Guion, "Cette obscure clarté". Des secrets et de leur dévoilement dans les Maximes", Littératures, automne 1998, n 39, p. 45-62 (surtout les p. 58-59).

9 Voir la notice et le catalogue du marquis de Granges de Surgères, Les portraits du duc de La Rochefoucauld [...], Paris, Damascène Morgand et Charles Fatout, 1882.

10 À la suite des Pensées de Madame de Schomberg sur les "Maximes" de La Rochefoucauld, dans François de La Rochefoucauld, Euvres complètes, Paris, Gallimard, coll. "Bibliothèque de la Pléiade", 1964, p. 713 [éd. crit. R. Kanters et J. Marchand]: "ce sont des phrases et des manières de parler qui sont plutôt celle d'un homme de la cour que d'un auteur". 
ministres du religieux qui continuent de monopoliser le discours philosophique ${ }^{11}$. Aussi, l'ambiguité que la critique a souvent vue dans les Maximes ou, si l'on préfère, leur duplicité résultent-elles de la conciliation d'une quête d'autonomie dans la manipulation du discours moral et d'une soumission à la répartition convenue des compétences dans les matières de ce même discours.

\section{Maximes en quête d'auteur}

L'anonymat des Maximes a biaisé leur réception par les contemporains: à travers le texte éclaté, les premiers lecteurs, qui, sollicités par Madame de Sablé, ne connaissent pas, pour la plupart, le nom de l'auteur, ont cherché à le démasquer, d'en fixer le portrait psychologique, moral et religieux. Le désinvestissement du locuteur est tel qu'il a exacerbé la fièvre de mettre un visage sur une prose qui se voulait anonyme, chez des lecteurs que dérouta l'effacement de l'auteur. C'est un visage et partant un nom, plus ou moins familier du fait des fréquentations de la société de cour, que les lecteurs cherchent. En cela le frontispice de l'édition de 1664 voit son allégorie dédoublée: l'arrachement du masque de Sénèque construit une scène doctrinale - les Maximes sont écrites contre les vertus des païens - qui détourne de l'irritante énonciation d'un texte sans visage. Le frontispice crée une illusion pas moins forte que celle de l'avis au lecteur ${ }^{12}$, sans parler du "Discours" d'Henri de La Chappelle-Bessé, qui performe toujours le même impérieux désir locutoire $^{13}$ : je vous veux là, mais ne m'y cherchez pas!

11 Voir Christoph Stroseztki, Rhétorique de la conversation. Sa dimension littéraire et linguistique dans la société française du XVII eiècle, Paris-Seattle-Tübingen, Papers On French Seventeenth Century Literature, coll. "Biblio 17", 1984, p. 120 et suivantes [trad. S. Seubert].

12 "En un mot, le meilleur parti que le lecteur ait à prendre est de se mettre d'abord dans l'esprit qu'il n'y a aucune des maximes qui le regarde en particulier, et qu'il en est seul excepté, bien qu'elles paraissent générales; après cela, je [c'est l'éditeur Claude Barbin, et non La Rochefoucauld, qui parle] lui réponds qu'il sera le premier à y souscrire, et qu'il croira qu'elles font encore grâce au cœur humain", dans Maximes suivies des Réflexions diverses, [...], Paris, Éditions Garnier, coll. "Classiques", 1983, p. 268 [éd. crit. J. Truchet]. Dans la suite de cet article, les citations des Maximes sont toutes faites à partir de cette édition.

13 Ce désir peut se relire, dans un contexte tout autre, à travers la lettre fameuse du duc à son fils François, quelques mois avant sa mort (op. cit., p. 658-660). 
Ainsi dépouillé du corps en sa visibilité sociale de bienséance, le texte des Maximes se donne pour désincarné; en retour, il appelle, dans sa manipulation, le désir d'une réincarnation. Pour exemple, le témoignage de Madame de Guyméné sur sa lecture première du manuscrit des Maximes:

Je n'ai encore vu que les premières maximes, à cause que j'avais hier mal à la tête; mais ce que j'en ai vu me paraît plus fondé sur l'humeur de l'auteur que sur la vérité. ${ }^{14}$

Le corps réinvesti n'est pas le tout de l'être physique: il est passé au crible des conventions - bumeur, ici, et air, ailleurs ${ }^{15}$ - qui sont le fait du corps dont l'exhibition est loisible - air ou manières - même si elle est sanctionnée lorsqu'elle tient des humeurs contraires à la civilité, à l'exemple emblématique de la mélancolie dénoncée par Nicolas Faret dans l'Art de la cour (1636). Il n'empêche, la réincarnation est ramenée avant tout vers la portée de l'énoncé, façon d'entériner combien le locuteur ne peut être identifié dans le tout de son corps et notamment, sans grivoiserie, dans son sexe, bien que tous les témoignages de l'enquête de Madame de Sablé parlent de l'auteur comme d'une personne masculine.

L'auteur qui est sous enquête est, en surcroît à son anonymat, un être double au sein duquel la face de représentation est délibérément choisie: c'est le stoïcisme exhibé de l'autoportrait de 1658.

Je suis peu sensible à la pitié, et je voudrais ne l'y être point du tout. Cependant, il n'est rien que je ne fisse pour le soulagement d'une personne affligée; et je crois effectivement que l'on doit tout faire, jusqu'à lui témoigner même beaucoup de compassion de son mal; car les misérables sont si sots que cela leur fait le plus grand bien du monde; mais je tiens aussi qu'il faut se contenter d'en témoigner et se garder soigneusement d'en avoir. ${ }^{16}$

14 Dans Euvres complètes, op. cit., p. 711.

15 Le doublet permet de classer objectivement les témoignages, entre ceux qui sont positifs dès lors qu'ils parlent de l'air de l'auteur ou de ses manières, et ceux qui sont plus réservés quand ils assignent à l'humeur la malignité du recueil; les premiers témoignages renvoient ainsi à la manifestation d'un éthos mondain; les seconds laissent sous-entendre un dérèglement psychopathologique rédhibitoire à la sociabilité mondaine.

16 Ibid., p. 5-6. On trouvera la même restriction au nom de la raison naturelle dans l'Entretien avec le chevalier de Méré sur la recherche du bonheur, dans 
Pareille rétractation dit l'héroïsme altier d'un cour qui se connaît et qui connaît les autres. Le portrait littéraire que La Rochefoucauld trace de lui-même est tout à une stylistique de la concession; cette caractéristique n'est toutefois pas un trait formel qui serait hétérogène à la matière, puisque c'est la même stylistique qui soustend le portrait que le cardinal de Retz trace de son ancien ennemi.

Il a toujours eu une irrésolution habituelle, mais je ne sais même à quoi attribuer cette irrésolution : elle n'a pu venir en lui de la fécondité de son imagination, qui n'est rien moins que vive; je ne la puis donner à la stérilité de son jugement, car, quoiqu'il ne l'ait pas exquis dans l'action, il a un bon fonds de raison: nous voyons les effets de cette irrésolution, quoique nous n'en connaissions pas la cause. Il n'a jamais été guerrier, quoiqu'il fût très soldat; il n'a jamais été par lui-même bon courtisan, quoiqu'il ait eu toujours bonne intention de l'être; il n'a jamais été bon homme de parti, quoique toute sa vie il y ait été engagé. Cet air de honte et de timidité que vous lui voyez dans la vie civile, s'était tourné, dans les affaires, en air d'apologie; il croyait toujours en avoir besoin: ce qui, joint à ses Maximes, qui ne marquent pas assez de foi en la vertu, et à sa pratique, qui a toujours été de chercher à sortir des affaires avec autant d'impatience qu'il y était entré, me fait conclure qu'il eût beaucoup mieux fait de se connaître, et de se réduire à passer, comme il l'eût pu, pour le courtisan le plus poli qui eût paru dans son siècle $1^{17}$.

L'irrésolution sans cause que le cardinal de Retz débusque dans son portrait de La Rochefoucauld n'est en fait que l'autre nom de la mélancolie; mais une mélancolie singulière, qui ne relève ni de l'abattement des facultés ni de la prostration du corps, et qui se trouve dans une communauté lointaine avec celle où le Problème $X X X, 1$ du Pseudo-Aristote situe l'origine du génie:

pour parler de mon humeur, je suis mélancolique, et je le suis à un point que, depuis trois ou quatre ans, à peine m'a-t-on vu rire trois ou quatre fois. J'aurais pourtant, ce me semble, une mélancolie assez supportable et assez douce, si je n'en avais point d'autre que celle qui me vient de mon tempérament; mais il m'en vient tant d'ailleurs, et ce qui m'en vient remplit de telle sorte l'imagination et m'occupe si fort l'esprit, que la

ibid., p. 729. À un siècle de distance, le souci du bonheur commande la même solution d'hypocrisie que celle que professera Diderot devant la maréchale de $\mathrm{B}^{* * * *}[$ roglie].

17 Ibid., p. 7. 
44

plupart du temps, ou je rêve sans dire mot, ou je n'ai presque point d'attache à ce que je dis. ${ }^{18}$

L'épanchement humoral de la mélancolie échappe à la simple caractérisation hippocratique du tempérament, dont l'appropriation est aussi un signe supplémentaire de distinction sociale au nom de la correspondance entre la supériorité du tempérament avec la supériorité de rang ${ }^{19}$ ou au nom d'un constat plus prosaïque et plus cinglant qui, avec les mots de Diderot, fera de la mélancolie une maladie de ventres trop pleins dans un XVIII ${ }^{\mathrm{e}}$ siècle où les "grands" seraient devenus "gras". Si, en plus de recevoir la liaison convenue entre passions et maladies, comme il le répétera dans ses Réflexions, La Rochefoucauld souscrit donc à une représentation convenue de la mélancolie, jointe à une justification sociale sous le sceau d'une communauté d'exception, il se rétracte dans le fait de remarquer au sein de sa complexion un surcroît d'afflux que singularise encore une source insituable. Convention assumée et spécificité revendiquée, qui met à distance la conformité première à la représentation sociale de la conscience aristocratique sous le couvert du tempérament mélancolique. Concession à la justification sociale et singularité inatteignable par le fait que sa source est atopique, pareil schème se relit dans les Maximes, lorsqu'il s'agit de définir de manière liminaire l'amour-propre:

Il est inconstant, et outre les changements qui lui ${ }^{20}$ viennent des causes étrangères, il y en a une infinité qui naissent de lui et de son propre fonds. ${ }^{21}$

Comme l'humeur mélancolique est excédentaire par un surplus que ne parvient à préciser l'autoportrait, la peinture de l'amourpropre le présente de même. Excédent et différenciation sans retour, l'amour-propre agit alors à hauteur de la définition de la bile par Galien que citera Jacques Esprit dans son traité miroir des Maximes: "La bile [...] n'est pas seulement différente des autres humeurs, elle l'est encore d'elle-même." ${ }^{22}$ Ainsi se nouent

18 Ibid., p. 4.

19 Corrado Rosso, Procès à La Rochefoucauld et à la maxime [...], Pise, Editrice Libreria Goliardica, "coll. Histoire et critique des idées", 1986, p. 70.

20 Je corrige à la suite de Paul Bénichou, "L'intention des "Maximes" ", dans L'écrivain et ses travaux, Paris, Librairie José Corti, 1967, p. 9.

21 Maximes, op. cit., p. 284.

22 Jacques Esprit, La fausseté des vertus humaines [...], Paris, Aubier, 1996, p. 87. 
dans une analogie serrée, autoportrait et Maximes, non tant dans leur thématique respective - la mélancolie, d'un côté, l'amourpropre, de l'autre - que dans ce qui est le principe même des deux textes: adhésion refusée de soi à soi et essence instable de l'être humain. L'analogie est d'autant plus serrée que c'est le même schème du surplus qui désigne - Jean Starobinski le note à la suite de l'exemple de Montaigne - le point de compétence du moraliste, comme un "surcroît de connaissance", avant qu'il ne se résolve - instabilité foncière du dire moral - en une "confession d'ignorance" 23 . Et si, dans son recueil de 1664, La Rochefoucauld n'a pas fait le portrait de la corruption de son esprit en cherchant les vices sous les vertus ${ }^{24}$, il y a laissé l'empreinte de sa mélancolie, au sens premier du terme, qui est le signe interchangeable de son "caractère" 25 et qui est le principe de la fusion refusée du dit de soi avec l'être qui le professe.

\section{La pose au miroir}

À la suite du jugement de Madame de Guyméné, la critique n'a pas manqué de relier le texte des Maximes au tempérament de leur auteur, ébauchant de la sorte une scénographie de l'énonciation mélancolique qui doit soit ouvrir à une lecture par rétractation - ce fut celle de Désiré Nisard ${ }^{26}$ —, soit en appeler à une mise en contexte qui fait écran à toute médiation qu'autorise une rhétorique des passions; la lecture des Maximes par Léon Levrault relève de cette dernière orientation où le critique invite le lecteur à se déporter dans la suggestion d'une scène qui tient à ce que le formalisme appelle la fonction imaginaire.

23 Dans "Complexité de La Rochefoucauld", dans Preuves, mai 1962, n 135 , p. 40.

24 C'est le désaveu souvent cité qui se trouve extrait de l'Entretien avec le chevalier de Méré, op. cit., p. 728: "[j]'ai remarqué [...] les défauts de l'esprit et du cour de la plupart du monde, et ceux qui ne me connaissent que par là pensent que j'ai tous ces défauts, comme si j'avais fait mon portrait".

25 Sur les liens métaphoriques et idéologiquement stabilisés de caractère et d'empreinte - c'est le même mot —, on se reportera à l'article de Françoise Berlan, "Le mot "caractère" dans Les caractères de La Bruyère", dans Les Champs du Signe, 1991, p. 121-142.

26 Histoire de la littérature française, Paris, Librairie Firmin Didot et Cie, 1889, p. 185 [16 éd.]: "Avant donc d'accepter les Maximes comme des vérités, il faut en ôter par la réflexion tout ce qui est inspiré de cette mélancolie dont La Rochefoucauld s'avoue atteint". 
Figurons-nous le duc, un soir de réception, dans le salon de la marquise, au milieu de presque tous les personnages dont nous venons de parler. Bien souvent, sans doute, avant que de se rendre à ces réunions littéraires, cet homme, qui avait si complètement gâché sa vie, s'était livré à des réflexions amères; et il en restait comme une ombre de profonde tristesse sur son front. 27

Différents dans leur visée, les protocoles de lecture de Nisard et de Levrault sont toutefois fort proches en ce qu'ils pointent, un peu au jugé, une antériorité 28 où se dessine la genèse des Maximes. Il y aurait alors l'antériorité du genre - la maxime avant La Rochefoucauld ${ }^{29}$ - mais cette avant-scène n'a d'intérêt que de souligner le contraste entre les avant-courriers et le recueil du duc; il ne reste plus guère, au sein de l'existence objective de l'auteur, que la scène fondatrice, celle de l'autoportrait, qui n'est telle que parce qu'elle subjugue l'événement traumatique de la défaite et de l'aveuglement causé par le coup de mousquet lors de la bataille du faubourg Saint-Antoine.

L'autoportrait construit une énonciation d'un je mélancolique ${ }^{30}$ dont procédera la rédaction même des Maximes $^{31}$. Pour leur première édition autorisée, elles se présentent dans le kaléidoscope de trois — sous bénéfice d'inventaire -

27 Maximes et portraits. Évolution du genre, Paris, Librairie classique Paul Mellottée, sd., p. 87 [1 $1^{\text {re }}$ éd. Librairie Delaplane, 1909].

28 Pas moins scène imaginaire de l'antériorité, cosmogonique cette fois, l'imprécation d'Alfred de Musset (Confession d'un enfant du siècle, Ve Partie, ch. IV) contre les esprits forts, qui veulent scruter au-delà des apparences pour n'y voir que le mal, rapporte au mot de Satan, au moment où il fut précipité en enfer, le principe créateur de la maxime, qui naîtrait d'une morosité dubitative et destructrice: Qui sait?

29 Exemple de cette antériorité exhumée, l'analyse en filiation de Hugues de Saint-Victor à La Rochefoucauld, en passant par la médiation culturelle d'Urbain Chevreau, que propose Vivien Thweatt, La Rochefoucauld and the Seventeenth Century Concept of the Self, Genève, Librairie Droz, coll. "Histoire des idées et critique littéraire", 1980.

30 Celle-ci déborde bien sûr les marques qui, pour être authentiques, sont celles de la physiologie mélancolique: "le teint brun", "les yeux noirs", "les cheveux noirs", plus ce "quelque chose de chagrin et de fier dans la mine" (CEuvres complètes, op. cit., p. 3).

31 Par cette orientation de la question, je me démarque résolument de Susan Read Baker ("La Rochefoucauld and the Art of the Self-Portrait", dans Romanic Review, vol. LXV, $\mathrm{n}^{\circ}$ 1, janvier 1974, p. 17) qui piste des liens thématiques entre l'autoportrait et le recueil. 
scénographies $^{32}$, où se réalise la médiation de l'œuvre au monde qui l'a vu apparaître. L'avis au lecteur de Claude Barbin, qui sera modifié dès la deuxième édition, le "Discours" de La ChapelleBessé, qui ne sera plus reproduit dans les éditions ultérieures, et enfin la grande maxime sur l'amour-propre qui disparaîtra aussi, sont ces trois scénographies où se joue l'énonciation et dont le traitement qu'elles subiront par la suite montre clairement le travail bien connu de l'effacement des marques énonciatives ou de leur neutralisation par la fusion du je en un nous consensuel par force. La performativité généralisante de la maxime tend en même temps à oblitérer les marquages historiques et spatiaux qui situeraient l'auteur dans le temps et le lieu de son énonciation. Chacune des Maximes se donne alors dans une parfaite autarcie, sans toutefois cesser de valider le lien qu'elle entretient avec le lieu de son énonciation biffée. Dans son autonomie, la maxime relève de la métaphore dont s'orne la première sentence supprimée: la peinture de l'amour-propre et le moraliste en effigie d'un peintre du tempérament et des vices. L'objet pictural anéantit, plus que tout produit symbolique, la topographie et la chronographie de sa genèse, en suscitant un temps et un lieu de la représentation tout autres. Mais au sein de la scénographie évidée des Maximes, demeure une force dialogique dont sont l'indice les modalités correctives ou atténuantes ${ }^{33}$, ainsi que l'ironie, qui suscite la connivence d'un savoir et d'une culture partagés dans le cadre élargi des entrelacs de la sociabilité où se rencontrent pensée religieuse, intérêts mondains et hexis aristocratique.

Poser "l'ambiguité référentielle du discours bref " 34 reste insatisfaisant en ce que pareille acceptation ne dépasse nullement la définition aristotélicienne de la sentence, ne brise donc pas l'enchantement paraphrasant de l'origine de la maxime, et en ce qu'elle scinde l'unité rhétorique et morale du phénomène par le fait d'une ambivalence rhétorique qui se mire dans les contrariétés morales. Ces contrariétés définissent le propre d'une éthique

32 Je reprends le concept à Dominique Maingueneau, Le contexte de l'œuvre littéraire. Énonciation, écrivain, société, Paris, Dunod, 1993, p. 121 et suivantes.

33 Corrado Rosso, op. cit., p. 175

34 Georges Molinié, "Statut énonciatif et fonction littéraire du discours bref à l'âge baroque: une figure de l'insatisfaction", dans Jean Lafond (dir.), Les formes brèves de la prose et le discours discontinu (XVIt-XVII siècles), Paris, Librairie philosophique J. Vrin, 1984, p. 90. 
mélancolique ${ }^{35}$ qui déstructure toute mimésis positive. Ainsi se comprend la pose du duc, se saisissant d'un miroir avant que de rédiger son autoportrait :

je viens de me tâter et de me regarder dans le miroir pour savoir ce qui en est, et je ne sais trop bien qu'en juger . ${ }^{36}$

Le but n'est assurément pas de tendre à une représentation plus vraie de soi - Jacqueline Plantié a montré les omissions volontaires des cicatrices du visage, moins par coquetterie que par souci d'effacer la faute politique qui a été cause de la blessure à la bataille du faubourg Saint-Antoine ${ }^{37}$. Mais, au-delà d'un souci politique d'amnésie volontaire, le geste prévient en fait une scénographie qui est directement inspirée de l'iconologie mélancolique ${ }^{38}$ et que certifie l'indécision finale du jugement sur soi. Il ne s'agit pas ici de poser les cadres d'une exposition de la vie vraie de La Rochefoucauld, mais plutôt de scruter l'articulation d'une expérience vécue, donc une expérience traumatique et fortement subjective, en rapport direct avec la représentation de soi.

\section{Le regard cicatrice ou la scénographie mélancolique des Maximes}

La blessure qui aveugle et dont le duc ne veut marquer la cicatrice dans son autoportrait est le trait physique de la genèse du discours moral, non tant du fait de l'analogie de l'âme avec le corps que du fait précisément de celle de l'omission des balafres avec l'effacement de l'énonciation. Le discours moral est réparateur jusque dans sa dénonciation du leurre thérapeutique.

Les défauts de l'âme sont comme les blessures du corps: quelques soins que l'on prenne de les guérir, la cicatrice paraît toujours [...] (M 194).

35 Sur l'ambivalence éthique de la mélancolie, voir Patrick Dandrey, "La rédemption par les lettres dans l'occident mélancolique (1570-1670). Contribution à une histoire de la jouissance esthétique", dans Marc Fumaroli, Philippe-Joseph Salazar et Emmanuel Bury (dir.), Le loisir lettré à l'âge classique, Genève, Librairie Droz, coll. "Travaux du Grand Siècle", 1996, p. 78.

36 Euvres complètes, op. cit., p. 3.

37 La mode du portrait littéraire en France 1641-1681, Paris, Honoré Champion, coll. "Lumière Classique", 1994, p. 263.

38 G. F. Hartlaub, Zauber des Spiegels. Geschichte und Bedeutung des Spiegels in der Kunst, Munich, R. Piper, 1951. 
La blessure des yeux aura été préliminaire à la mélancolie; elle re-présente l'histoire mythique du dieu tutélaire des esprits chagrins. Aveugle, le moraliste est mutilé d'une manière qui évoque celle par laquelle le dieu Saturne-Cronos, "procréateur de toutes choses"39, a été castré par son fils Jupiter-Zeus, appliquant la même loi dont son père avait frappé son propre géniteur. La Théogonie d'Hésiode débrouille la réversibilité ${ }^{40}$ de la blessure: du sexe tranché d'Ouranos, naît Aphrodite; l'ambivalence foncière de Saturne célèbre tout à la fois le dieu des moissons de l'Âge d'Or et la divinité stérile. Relevant de cette ambivalence mythique, l'œil blessé devient plus aigu pour avoir fait l'expérience traumatique de ce que Locke ${ }^{41}$ nommera plus tard la tache aveugle, et de la réversibilité de la force du réel sur la conscience occulaire qui le contemple et le juge. C'est l'expérience du 2 juillet 1652, lorsque le duc reçut de biais "une mousquetade qui, lui perçant le visage au-dessous des yeux, lui fit à l'instant perdre la vue " ${ }^{2}$. Au témoignage des Mémoires de la Grande Mademoiselle ${ }^{43}$, La Rochefoucauld perdit dans l'accident, en plus de la vue, la parole. Ainsi, le duc subit la double mutilation contraire au principe même du discours moral dont il sera le tenant après la Fronde. La guérison se manifestera dans le distinguo implicite

39 Raymond Klibansky, Erwin Panofsky et Fritz Saxl, Saturne et la mélancolie. Études historiques et philosophiques [...], Paris, Gallimard, coll. "Bibliothèque Illustrée des Histoires", 1989, p. 211 [trad. F. Durand-Bogaert et L. Evrard].

40 Voir Ernest Pascal, "Le mythe de l'origine du pouvoir dans la Théogonie", Cabiers d'études anciennes, 1990, vol. XXIII, Hommage à la mémoire d'Ernest Pascal, t. 1, p. 20, où le philologue synthétise la logique du mythe — de tout mythe grec — comme "développement de principes ambigus en contradictions" les uns avec les autres, aussi longtemps que le mythe demeure dans le temps anhistorique. Cette lecture se troue vérifiée a contrario par les effets du syncrétisme de Cronos et de Saturne; le mythe du dieu castré rencontre alors l'histoire et l'espace des croyances humaines qui fondent le dieu grec avec le dieu latin des moissons par une légende rapportée sur le mythe: détrôné par Zeus, Cronos se serait retiré en Italie où il se serait vu nanti des attributions propres au dieu Saturne, retrouvant les prospérités qu'Hésiode lui attribuait avant l'attentat (voir cette légende rapportée dans le Dictionnaire abrégé de la fable [...], par Chompré, Neuchâtel, 1794, à l'article Saturne: "[c]e fut-là [en Italie] qu'il enseigna l'agriculture aux hommes, \& le temps de son règne fut si heureux, qu'on l'appella l'Age d'or").

41 Essai sur l'entendement humain, Livre I, ch. I.

42 Mémoires, dans Euvres complètes, op. cit., p. 205; je souligne.

43 Cité dans ibid., p. 802: "je m'arrêtai pour parler à lui; mais il ne me répondit pas: c'était tout ce qu'il pouvait faire que d'entendre". 
du moraliste, qui y trouve la légitimité de sa parole, et des hommes, à qui il entend parler, dans la maxime supprimée sur l'amour-propre: "cette obscurité épaisse qui le cache à lui-même, n'empêche pas qu'il ne voie parfaitement ce qui est hors de lui, en quoi il est semblable à nos yeux, qui découvrent tout, et sont aveugles seulement pour eux-mêmes " 44 .

Au témoignage de Gourville, cette fois-ci, La Rochefoucauld blessé plaça au-dessous du portrait gravé de sa maîtresse, Madame de Longueville, ce distique:

Faisant la guerre au roi, j'ai perdu les deux yeux;

Mais, pour un tel objet, je l'aurais faite aux Dieux. ${ }^{45}$

Par la brièveté du verset se trouve condensé un saut mythologique, que la psychanalyse ramène à une même phobie: perte de la vue et castration. Dans la sanction de l'bybris, Saturne cède devant CEdipe, mais un CEdipe moderne, dans le prisme d'Hamlet ${ }^{46}$, c'est-à-dire mélancoliquement prostré dans le sentiment de la faute et acculé aux fausses justifications. Et c'est sans doute là que se comprend, dans le filtre non de la psychanalyse mais de la philosophie, l'effet d'antipathie des Maximes dès lors que l'auteur odipien se refuse les voies normalisantes de l'adhésion aux lois sociales, autrement que par l'hypocrite paraitre, double face de l'héroïsme de la maxime 206 qui célèbre la réversibilité occulaire: "C'est être véritablement honnête homme que de vouloir être toujours exposé à la vue des honnêtes gens".

L'CEdipe se donne pour ininterrompu: les Mémoires ne laisseront entendre aucune saisie des enjeux de la Fronde comme une quête d'une communauté large d'intérêts qui dépasseraient enfin les égoïsmes partisans, clientélistes, ou personnels; les Maximes déploieront la scène de l'égoïsme violent, sous les auspices d'un locuteur hostile aux stratégies de la bienveillance comme loi d'une morale pratique de la vie en société. Ce qui mettrait un terme à la violence de l'Edipe, ce serait - ici - l'acte critique que sanctionne toute maxime, mais il ne tient que dans l'espace circonscrit de la sentence. Hors d'elle, il apparaît d'une pertinence et d'une efficacité illusoires tant par l'estompement de l'énonciation que

44 Maximes, op. cit., p. 283-284.

45 Euvres complètes, op. cit., p. 802.

46 Voir, à la suite des Leçons d'introduction à la psychanalyse de Freud (XXI ${ }^{\mathrm{e}}$, Paul-Laurent Assoun, Littérature et psychanalyse, Paris, Ellipses, coll. "Thèmes \& Études", 1996, p. 90. 
par le fait que les Maximes dénient formellement toute sociabilité: face à je qui se dérobe, il ne peut y avoir de contrat avec le vous - ni avec le nous de feinte - de la collectivité dont les représentations et les pratiques de pacification, toutes à l'artifice, ne tiennent pas face à la force apocalyptique d'une nature obscure par les passions.

Il semble que la nature ait caché dans le fond de notre esprit des talents et une habileté que nous ne connaissons pas; les passions seules ont le droit de les mettre au jour, et de nous donner quelquefois des vues plus certaines et plus achevées que l'art ne saurait faire (M 404).

Pareille croyance, quasi primitiviste, renoue avec les institutions mythiques qui font échoir à des forces occultes des droits que la raison classique leur dénierait. Voilà l'ultime stratagème d'CEdipe qui perdure violent par son inaptitude "à engendrer des liens sociaux positifs, harmonieux et stables" ${ }^{47}$. Enthymème tronqué, la maxime fait sens à la violence oedipienne qui se refuse les voies de la dialectique. Madame de Schomberg l'avait pressenti :

après la lecture de cet écrit, l'on demeure persuadé qu'il n'y a ni vice ni vertu à rien, et que l'on fait nécessairement toutes les actions de la vie. ${ }^{48}$

Sur ce pessimisme qui couronne l'amour-propre, les passions et, dans l'ordre de la contingence, la fortune dans un déterminisme quasi sauvage, la dialectique du lien s'efface devant l'analogie allusive, dernier reliquat d'une intelligence des choses et des êtres, cet autre nom de la condition minimale de la vie en société dont rien ne soutient les chances d'une pacification.

Réversibilité de l'CEdipe, après celle de Saturne, tous deux frères en castration: le commentaire de seconde main de l'un des lecteurs du manuscrit des Maximes replace l'effet du recueil comme un dévoilement impudique qui blesse la vue.

J'en parlai hier à un homme de mes amis, qui me dit qu'il avait vu cet écrit, et qu'à son avis, il découvrait les parties honteuses de la vie civile et de la société humaine, sur lesquelles il fallait tirer le rideau: ce que je fais, de peur que cela fasse mal aux yeux délicats [...]. ${ }^{49}$

47 Mikkel Borch-Jacobsen, Le sujet freudien, Paris, Flammarion, coll. "La philosophie en effet", 1982, p. 242.

48 Dans Euvres complètes, op. cit., p. 713.

49 Ibid., p. 716. 
Si l'antipathie des Maximes est le signe passionnel d'un CEdipe sans terme dans sa violence ${ }^{50}$, le recouvrement de la vue est parallèle à la rémission mythique de Saturne: déchu, le vieux roi des dieux porte désormais les emblèmes redoutables du temps; le regard, devenu celui du moraliste, se charge du faix de la mélancolie qui se masque d'autant plus qu'elle est due à une omission, celle des cicatrices balafrant le visage du duc.

\section{La houle de l'océan et les métaphores des passions mélancoliques}

Patrick Dandrey a brillamment balisé les lignes de faîte Montaigne, Shakespeare et Cervantès - d'une rhétorique où l'analogie entre la genèse du texte et la mélancolie faisait de la seconde le principe créateur de l'œuvre mais aussi celui de sa réception, oscillant alors, dans le prisme ancien du livre comme nourriture de l'âme, entre ingestion ravie et régurgitation écourée $^{51}$ - c'est là aussi, pour partie, la réception des Maximes. Et de citer aussi Jean-François Sarasin qui place son premier Discours poétique ${ }^{52}$, sous le signe du jeu de miroir entre l'océan, son âme et son inspiration mélancolique, sollicitée par l'élément marin qui ne parviendra à s'imposer à une muse désordonnée qu'au terme de l'expérience des contraires, tel l'épanchement ambivalent de la mélancolie qui fait hésiter entre exaltation créatrice et prostration morose.

Au bord de l'Océan, où le flot qui se joue

Avec beaucoup de bruit produit un peu de boue,

50 C'est ce que j'entends montrer dans une communication lors du colloque international: De la sympathie sous l'Ancien Régime. Discours, savoirs, sociétés (les actes paraitront, sous la dir. de Thierry Belleguic, aux Presses de l'Université Laval).

51 "La rédemption par les lettres", loc. cit., p. 63 et suivantes. À partir de ce faisceau, peut se relire alors de manière plus heuristique qu'anecdotique l'échange entre le duc et Madame de Sablé, échange de maximes contre bons potages et autres délicatesses gastronomiques.

52 Que la poésie lui sert de divertissement (dans l'édition posthume des Nouvelles ouvres [...], Paris, Claude Barbin, 2 vol., 1674). Reproduit par Patrick Dandrey, ibid., p. 84, n. 30 - il manque visiblement un vers. C'est une scénographie mélancolique que déploie encore la chanson "Charme secret des maux les plus puissans", alors qu'elle fait défaut dans l'élégie "Le mélancolique", si ce n'est comme blason inséré à l'instant d'évoquer Don Quichotte (dans Euvres de Monsieur Sarasin, Rouen-Paris, Augustin Courbé, 1658, respectivement, p. 78 et p. 100-103). 
Je flatte ma tristesse à composer des vers,

Et laisse en liberté couler ma poésie

Selon les mouvements où va ma fantaisie.

Tantôt je suis moral, tantôt je suis d'amour,

Je peins une naïade, un jardin, un beau jour,

Un étang, des rochers, des forêts, une source,

Le lit où le soleil s'en va finir sa course,

Et tout ce que je vois qui s'offre à mon esprit

Avec facilité ma Muse le décrit.

Reprenant le stéréotype baroque de la "mer très amère "53, la houle des flots fédère en une même image le bruit du monde en un lieu qui le nie, à l'instar de l'ermitage charentais de Guez de Balzac, les aléas de la fortune saturnienne, qui a violenté un autre écrivain de la mélancolie, Tristan l'Hermite, dans le souvenir chagrin d'une adolescence disgraciée, si ce n'est encore, dans une inspiration commune aux deux poètes baroques, l'effet de la fièvre mélancolique qui rend intermittents la muse et le discours qu'elle inspire ${ }^{54}$. Le ressac de l'océan deviendra, dans la seconde moitié du $\mathrm{XVII}^{\mathrm{e}}$ siècle, le creuset homologique de la conversation pure par la métaphore du flux et du reflux pour tracer le lieu du dialogue de la parfaite urbanité, à l'instar de la topographie du bel esprit qui ouvre les Entretiens d'Ariste et d'Eugène.

Dans sa conclusion, la grande maxime supprimée sur l'amourpropre cercle et clôture la scénographie du recueil, qu'avait ouverte l'avis de Claude Barbin par le sésame en forme de déictique: "Voici un portrait du coeur humain [...]", auquel répond: "Voilà la peinture de l'amour propre." 55 Mais cette clôture est ouverture de la scénographie de la mélancolie par les ornements métaphoriques de la mer rapportés à l'amour-propre ${ }^{56}$ :

53 L'expression est de Jacques de Billy. Voir, entre autres, Alain Corbin, Le territoire du vide. L'Occident et le désir du rivage 1750-1840, Paris, Flammarion, coll. "Champs", 1990, p. 19.

54 Extrait de l'élégie qui clôt quasi le Page disgracié (Paris, Gallimard, coll. "Folio", 1994, p. 261 [éd. J. Prévot]) : "Mon esprit s'égara de discours en discours. / Tantôt je croyais être en la troupe des Anges, / Et là de mon Sauveur exalter les louanges; / Tantôt je croyais être au plus creux des Enfers, / Tout embrasé de feux et tout chargé de fers; / Le plus brillant objet à mon oil était sombre, / Et même la clarté me paraissait une ombre". La fusion de l'élément marin avec la mélancolie est plus sereine dans la Mer (1627), mais elle n'en maintient pas moins la scénographie de la tristesse douloureuse.

55 Respectivement, p. 267 et 285 dans Maximes, op. cit.

56 La comparaison est une topique du discours religieux sur l'amour-propre, comme le montre Jean Lafond ("L'amour propre de La Rochefoucauld (MS 1). 
la mer en est une image sensible, et l'amour-propre trouve dans le flux et le reflux de ses vagues continuelles une fidèle expression de la succession turbulente de ses pensées, et de ses éternels mouvements.

Scénographie délibérée par l'ostentation de la liaison analogique — "une image" —, la péroraison inscrit un déplacement double: l'éveil nouveau des sens et la délimitation d'un nouvel espace pour le sentir autobiographique qui se dira dans le schème binaire du flux et du reflux, qui enserrera la vie vécue dans le lacis d'une permanence dialogique, qui fera de la maxime la quintessence d'une écriture fermée du moi, extrapolé de son existence et ramené à l'expression intemporelle d'une sentence dont le peaufinage d'édition en édition, de 1664 à 1678, est la dernière trace de la durée vécue.

\section{La genèse mélancolique des Maximes}

Il n'est que trop évident que le portrait bien connu du moraliste que Sainte-Beuve tracera plus tard est directement inspiré des lignes concourantes de l'autoportrait et du texte du cardinal de Retz.

Avec ces diverses qualités essayées de guerrier, de politique, de courtisan, il n'était dans aucune tout entier; il y avait toujours un coin essentiel de sa nature qui se dérobait et qui déplaçait l'équilibre ${ }^{57}$

Ainsi restituée dans l'équilibre instable ${ }^{58}$ de l'être avec lui-même, avec les choses et avec les événements, l'image du moraliste se donne comme un portrait aux Vanités, auquel répondrait, dans le prisme anachronique du toedium vitce romantique, le portrait du duc par Devéria, gravé par A. Lalauze à la fin du XIX ${ }^{\mathrm{e}}$ siècle et

Histoire d'un thème et d'une forme", dans L'homme et son image. Morales et littérature de Montaigne à Mandeville, Paris, Honoré Champion, coll. "Lumière Classique", 1996, p. 106.

57 Portraits de femmes, dans Euvres, Paris, Gallimard, coll. "Bibliothèque de la Pléiade", vol. II, 1951, p. 1250 [éd. crit. M. Leroy].

58 Voir Marie-Claude Lambotte, "La destinée en miroir", dans Alain Tapié (dir.), Les vanités dans la peinture au XvII siècle. Méditations sur la richesse, le dénuement et la rédemption, Paris - Caen, Albin Michel - Musée des BeauxArts de la Ville de Caen, 1990, p. 31. Jean Starobinski a analysé l'effet de déséquilibre produit par les Maximes, dans "Complexité de La Rochefoucauld", loc. cit., p. 38-39. 
inspiré du dessin de Pierre-Philippe Choffard qui reproduisit le second portrait en émail par Petitot pour accompagner l'édition des Maximes par l'imprimerie du Louvre en $1778^{59}$. Le portrait de Devéria montre la rêverie du duc, représenté de trois quarts, revêtu de l'armure, ceint d'une vaste écharpe, assis à l'ombre d'un arbre, le menton dans la main — geste mélancolique par excellence; enfin, il saisit l'instant critique de la vie du duc, songeur dans la défaite que symbolise le casque posé au pied du rocher. Le coude gauche écrase sur la cuisse gauche une feuille de papier dont ne sont lisibles que les six dernières lettres d'un titre qui se reconstituent dans le savoir des initiés: Réflexions morales.

Jean Starobinski saisit cet instant critique pour en faire le point de césure "entre le temps de l'illusion et le temps de la désillusion", entre "l'action, d'abord, puis, faute de mieux, la réflexion et la littérature " 60 . Les Maximes ouvrent alors aux analogies invérifiables de la spécularité: réflexion de la conscience meurtrie qui fait réfléchir à son tour et qui exhibe moins le vrai visage de Sénèque qu'elle ne présente à son lecteur un miroir vrai. De la sorte, les Maximes recomposent l'iconologie de la mélancolie au miroir, qui n'est pas moins présente dans la littérature que dans la peinture, à l'exemple de l'ouverture du Page disgracié où Tristan L'Hermite s'offre à la relation nostalgique de son adolescence dans l'irrésolution du dessein de répondre au désir de l'Autre - Thirinte — dans la rétention d'une histoire échouée et dans le protocole de la vérité d'un texte déceptif: "[c]'est une fidèle copie d'un lamentable original, c'est comme une réflexion de miroir " ${ }^{61}$. Mise en mots de l'iconologie mélancolique, le Page disgracié l'est encore à l'instar d'un point commun qu'il partage avec les Maximes: auteur au miroir de luimême, Tristan L'Hermite fait de sa pose le principe de son texte, qui sera celui de l'amour-propre. C'est explicitement au nom de l'amour de soi que les histoires écossaises, rapportées et hétérogènes au projet autobiographique, sont intégrées dans le texte ${ }^{62}$.

59 Voir la notice et le catalogue, déjà cités, du marquis de Granges de Surgères, p. $8-9$ et 38.

60 Dans son introduction aux Maximes et Mémoires, op. cit., p. 23.

61 Le page disgracié, op. cit., p. 24.

62 Ibid., p. 161-162: "Pendant le peu de jours que nous fûmes ensemble, ce jeune seigneur disgracié me conta beaucoup d'aventures de guerre, et parmi cela quelques histoires d'amour dont la fin était déplorable, et c'étaient des matières qui répondaient à ma fortune; [...]. Les cœurs blessés en même 
56

Le duc, qui, stö̈cien au milieu de sa guerre à Sénèque ${ }^{63}$, ne veut rien laisser paraître de ses sentiments, diffère certes du petit écuyer, mais ils se retrouvent tous deux dans l'effet de leur complexion, le premier souffrant d'un surcroît humoral, le second meurtri au siège de ses passions par l'arrachement du cœur qui est la conclusion nostalgique de la remémoration de sa "glorieuse servitude" auprès d'une jeune Anglaise. L'expérience vécue est somme toute la même: pour La Rochefoucauld, celle d'un échec politique, et, pour Tristan L'Hermite, celle d'une errance saturnienne ou d'un exil, en lieu et place d'un voyage, sous le signe de Mercure et des origines ${ }^{64}$, qui ne fut jamais fait et dont la nostalgie vient attenter au bonheur trop fastueux pour être durable des amours et à l'enlisement dans la volupté, ce vice qui a fait obstacle à l'initiation que le philosophe chimiste avait promise au page. Le voyage d'éducation, béance textuelle en anamorphose du vide intérieur de l'auteur, aurait commencé par le commencement d'une ascèse chrétienne: un pèlerinage sur le tombeau du Christ à Jérusalem. Vingt ans avant les Maximes, Tristan L'Hermite avait cherché la voie d'un dire vrai de soi dans le temps critique où les discours se voyaient irrémédiablement répartis en deux pôles d'inspiration: les ornements mythologiques devaient être réservés à la fable antique et l'inspiration chrétienne, aux ouvres de l'histoire sainte et aux discours plus ou moins fictifs du présent ${ }^{65}$. Homologique d'une conscience divisée entre les amertumes vécues et les aspirations perdues, l'empyrée du Page disgracié est déchiré entre la malignité des astres et de la Fortune et le Ciel miséricordieux et providentiel. Pour dire la désillusion dans le présent de son temps,

endroit sont comme les luths qui sont accordés à même ton: l'on ne saurait toucher une corde en l'un qu'on ne fasse branler celle qui lui répond en l'autre; l'on voit ainsi les affligés compatir facilement au malheur d'autrui, et cette émotion vient de ce ressort qu'on appelle amour de nous-mêmes ".

63 Ce paradoxe a souvent été relevé et Susan Read Baker (Collaboration et originalité chez La Rochefoucauld, Gainesville, University Presses of Florida, coll. "Humanities Monographs", 1980, p. 25 et suivantes) l'explique par le menu.

64 Le page est né dans le cycle de Mercure. Sur l'opposition Saturne et Mercure, abondamment exploitée par les alchimistes, voir notamment le Livre d'Abraham, fo 1, Bibliothèque de l'Arsenal (Paris), ms 3047. Tristan L'Hermite ne fait que reprendre le divorce fondateur entre "complexion naturelle" et mélancolie circonstancielle où Montaigne (Essais, II, 8) avait posé l'origine de son projet d'écriture.

65 C'est pour le moins le résultat de la querelle faite à Daniel Heinsius par Guez de Balzac, en 1636, et reprise, en 1643, par Claude Saumaise. 
l'auteur classique n'a plus le refuge des oripeaux de l'érudition païenne et faire entendre une représentation de soi sous le sceau de l'échec est pour le moins une entreprise subversive au sein de la doxa chrétienne pour laquelle l'individu ne s'appartient pas puisqu'il soumet sa destinée à un projet qui le dépasse. Impie, le discours de l'échec n'adhère plus aux conventions esthétiques qui refusent le syncrétisme de l'odyssée d'Ulysse avec l'autobiographie d'une existence triviale que ne rehaussent des projets glorieux comme autant de chimères ${ }^{66}$. Produit de la mélancolie, le Page disgracié résulte aussi des tensions que la doxa chrétienne n'a cessé d'entretenir autour de l'humeur noire; les Maximes seront de ce point de vue plus fardées par l'écran qui médiatise l'évidence du texte et le lieu peccable de sa genèse. Toutefois, elles n'en demeurent pas moins quête de l'origine ${ }^{67}$ — celle de l'amourpropre -, quête substitutive d'un projet autobiographique qui, par la maxime, est possible dans le présent éthique de son auteur: en même temps qu'elle s'énonce, rapportée à la matrice de l'amourpropre, la vie dit déjà sa fin puisque ne peut être surpassé le principe premier, sinon guerrier, de toute existence humaine, l'égoïsme, ce nom barbare de l'instinct de vie et, paradoxe levé par les Maximes, du grégarisme des lâches.

\section{Le Speculum perdu}

La maxime entérine l'éclatement du sens ${ }^{68}$; elle recueille les miettes de l'édifice dogmatique qui, construit dans le rais du regard divin, a été rabaissé à un horizon sans perspective et à une multiplicité des points de vue avec le renversement de l'ordre théologique en un ordre omnicentrique ${ }^{69}$, auquel ont conspiré, dans la tradition prestigieuse de la philosophie, Cicéron, Nicolas de Cuse, Giordano Bruno et Montaigne, qui avait ramené la sagesse céleste à la dimension de l'homme ${ }^{70}$, ou, dans le cadre

66 Dans la dernière partie de sa thèse sur La Rochefoucauld, Jean Lafond a très justement rapporté l'ironie des Maximes à la difficulté de faire entendre un pessimisme en une solution univoque ou en filiation homogène des traditions, soit modernes, voire mondaines, soit antiques, sinon augustiniennes.

67 Jean Starobinski, Le remède dans le mal. Critique et légitimation de l'artifice à l'âge des Lumières, Paris, Gallimard, coll. "N.R.F. Essais", 1989, p. 69.

68 Voir Ralph Heyndels, La pensée fragmentée. Discontinuité formelle et question du sens [...], Liège-Bruxelles, Pierre Mardaga, coll. "Philosophie et Langage", 1985, ch. II.

69 Voir la lecture de Corrado Rosso, op. cit., p. 147-149. 
58

des événements contemporains du moraliste, la crise de la scolastique. Redondance d'un savoir spécialisé sans cesse revisité et d'une actualité à laquelle les amis jansénistes apportent leur concours destructeur - ce sera la Logique de Port-Royal ou les Pensées de Pascal —, l'œil humain ne saisit plus la perspective de son regard que comme une interrogation sans réponse. Il y a un manque au sein du syllogisme universel. Comme dans son autoportrait qui scinde l'ordre de l'esprit et celui du coeur, de même qu'en toute cohérence, La Rochefoucauld se refuse à se persuader de l'intime correspondance de l'apparence physique avec l'intériorité du caractère, les Maximes relèvent - Aristote le pointait déjà ${ }^{71}$ — d'une analogie tronquée. C'est en cela surtout que se réalise le passage du portrait à la maxime: tout deux renvoient à une même dialectique avortée.

En déshérence de la totalité, la maxime la reconquiert par les voies mélancoliques de l'errance temporelle qui a succédé à la saisie transcendante du sens en un hors temps où se produit la compréhension immédiate et transfigurante. Dans le souvenir perdu du geste de Josué, la maxime convie à l'expérience lointaine et nostalgique du Tout par les chemins dispersés d'un recueil, mouvant d'édition en édition; et, si elle se refuse au tout dire du Tout, elle s'en approche au terme de l'inlassable reprise d'une proposition qui, pour n'être totale, est finalement toujours la même, égrenée aux accents mondains de la variété ${ }^{72}$. Plusieurs liens métaphoriques, dans la production du duc comme dans celle de ses contemporains, rendent compte de ce travail de peaufinage; ils se présentent en fait comme le substrat divers d'une quête de la totalité reconquise par la magie du tour analogique qui relèvera de la métallurgie ${ }^{73}$ de l'essai critique

70 Louis van Delft, "Du théâtre du monde à l'anatomie", dans Claire Carlin (dir.), op. cit., p. 59.

71 Rhétorique, 1394a: "comme les enthymèmes sont des syllogismes qui portent sur telle ou telle chose, presque toujours les conclusions des enthymèmes et leurs points de départ, abstraction faite du syllogisme, sont des sentences".

72 La Bruyère l'avait noté : "cette unique pensée, comme multipliée en mille manières différentes, a toujours, par le choix des mots et par la variété de l'expression, la grâce de la nouveauté" (Discours sur Théophraste, dans Les caractères de Théophraste traduits du grec [...], Paris, Éditions Garnier, coll. "Classiques", 1962, p. 15 [éd. crit. R. Garapon]).

73 Porosité analogique que permet encore l'Encyclopédie de Diderot et de d'Alembert où essai désigne autant le genre initié par Montaigne que l'"examen d'un minéral". 
pour les Réflexions, ainsi que l'entérine La Fontaine dans son "Discours à monsieur le duc de La Rochefoucauld" ${ }^{74}$, ou qui, plus instable dans le cas des Maximes, prendra tout autant à la numismatique qu'à la chimie :

Il semble que l'amour-propre soit la dupe de la bonté, et qu'il s'oublie lui-même lorsque nous travaillons pour l'avantage des autres. Cependant c'est prendre le chemin le plus assuré pour arriver à ses fins; c'est prêter à usure sous prétexte de donner; c'est enfin s'acquérir tout le monde par un moyen subtil et délicat (M 236).

En vue d'une économie morale ${ }^{75}$ où la maxime se repense dans les liens avec la médaille dont Louis Marin a montré la valeur de moniment ${ }^{76}$, l'amour-propre et, partant, le discours qui prétend en rendre compte passent par le moyen d'une chimie des mots ${ }^{77}$, où les contemporains n'ont pas été longs à jouer de l'homonymie.

\section{La chimie des maux ou l'alambic de la phrase}

Dans un commentaire ironique, un lecteur anonyme du manuscrit que fit circuler Madame de Sablé situe la rédaction des Maximes dans le réseau métaphorique d'une chimie des remèdes et des poisons, après avoir essayé celle de l'architecture pour rendre compte de la forme discontinue de l'ouvrage:

je n'entends rien en ces choses si subtiles et si délicates; [...] ce n'est qu'une collection de plusieurs livres d'où l'on a choisi les sentences, les pointes et les choses qui avaient plus de rapport au dessein de celui qui a prétendu en faire un ouvrage considérable. [...] Il y a beaucoup de simples [livres] dont le suc

74 Fable 14 du Livre X, v. 58: "Vous qui m'avez donné ce qu'il [le discours] a de solide", en cohérence avec "les esprits corps, et pétris de matières", dont le poète a voulu parler, renvoie, de ce fait, moins aux Maximes qu'aux Réflexions.

75 Je me permets de me réserver d'en faire la démonstration plus complète ailleurs, à partir du "Discours" de La Chapelle-Bessé et de l'article "Intérêt" de Saint-Lambert dans l'Encyclopédie.

76 Louis Marin, Le portrait du roi, Paris, Éditions de Minuit, "Le Sens Commun", 1981, p. 150 et suivantes.

77 Selon la judicieuse analogie de Roland Barthes, "La Rochefoucauld, Réflexions ou sentences et maximes", dans Le degré zéro de l'écriture, Paris, Éditions du Seuil, coll. "Points", 1972, p. 83. 
60

est poison, qui ne sont point dangereux lorsqu'on n'en a rien extrait et que la plante est en son entier. ${ }^{78}$

Plus de quinze ans après la dernière édition des Maximes, le Père Bouhours présentera l'ouvrage de Jean Corbinelli, les Anciens historiens latins réduits en maximes. Ce qui s'y relit, dans le triomphe du bel esprit, c'est la reconnaissance du recueil du duc sous la figure riante d'un Héraclite qui aurait été substitué à un Démocrite, qui n'aurait pas moins été "un homme du monde" et à qui aurait manqué "un talent particulier pour egayer \& pour animer la conversation". Dans les mots du père jésuite, se retrouve encore, par les stéréotypes définissant l'excellence du lettré, l'heureuse association de l'érudition et de la politesse, du génie profond et des manières du monde qui donne au recueil "un air qui ne sent ni le College ni l'Academie" "79. Face gaie d'un même procédé et d'une même poétique du déliement sans ordre, le livre de Corbinelli répond pleinement à la rencontre sereine, voire frivole, des deux finalités des belles-lettres classiques - le placere et le docere - en n'exigeant qu'une attention peu soutenue et ne cessant "d'instruire en amusant". Un tel résultat vient d'une opération subtile, au sens chimique qu'il avait au $\mathrm{XVII}^{\mathrm{e}}$ siècle, sur la fréquentation assidue de l'histoire romaine:

en lisant les Historiens il s'est avisé de remarquer les Maximes qui y sont répanduës, \& de tourner même en Maximes certains passages qui n'ont rien que d'historique: c'est-à-dire, si j'ose m'exprimer de la sorte, qu'il a mis ces Passages à l'alambic; \& que chaque Maxime Françoise est comme l'essence \& l'esprit du Passage latin qui la suit. Ainsi, sa veuë principale n'a pas été de traduire fidellement les endroits à quoi il s'est attaché; mais d'en extraire quelque chose de moral ou de politique selon le sens qu'ils renferment.

Par sa formulation réticente, Bouhours laisse entendre non tant l'audace de son image que le recours à un mot spécialisé et dont

78 Euvres complètes, op. cit., p. 715.

79 Avertissement de Dominique Bouhours en tête de Jean Corbinelli, Les anciens bistoriens latins reduits en maximes. [...], Paris, Georges et Louis Josse, 1694, np. À comparer avec le témoignage anonyme reçu par Madame de Sablé lors de sa consultation sur le manuscrit des Maximes: "L'on voit bien que ce faiseur de maximes n'est pas un homme nourri dans la province, ni dans l'Université; c'est un homme de qualité qui connaît parfaitement la cour et le monde, qui en a goûté autrefois toutes les douceurs, qui en a aussi senti souvent les amertumes, et qui s'est donné le loisir d'en étudier et d'en pénétrer tous les détours et toutes les finesses" (Euvres complètes, op. cit., p. 718). 
le dérivé verbal paraît en complète désuétude à la fin du $\mathrm{XVII}^{\mathrm{e}}$ siècle ${ }^{80}$. Un grammairien averti ne peut l'ignorer et, s'il passe outre à des scrupules de norme, c'est que la comparaison de la production de la maxime et de la distillation par l'alambic est exacte et que, rapportée au projet de Bouhours, la sentence exalte le purisme qu'il voulut imposer aux belles lettres.

L'image de l'alambic, instrument d'une curiosité allodoxe dans la société aristocratique, rend parfaitement la foncière paratopie ${ }^{81}$ du moraliste classique, qui issu des salons, tend à se déplacer dans le non lieu des vérités intangibles. En même temps, elle commande de mettre au jour la trivialité du projet des Maximes par le fait qu'elle désymbolise les métaphores, rendues alors au sens premier des choses dans un discours qui n'est fait que de matière, épanchement humoral en son origine. Au moment de clore son histoire de la maxime et du portrait, que leur coïncidence historique détermine à former un genre unique, Léon Levrault jouera des mêmes analogies métaphoriques dans une péroraison qui se perd dans une histoire littéraire qui n'est plus qu'olfaction d'un passé supposé révolu ${ }^{82}$ : "on ne veut plus du petit flacon de cristal d'où tombait, goutte à goutte, l'essence subtile de la pure morale" 83 . Les essences, pour être peut-être trop capiteuses ou entêtantes, sont oubliées dans ce musée par excellence des nostalgies qu'est le cabinet imaginaire des parfums ${ }^{84}$. Par sa physique du feu, la distillation à l'alambic est directement métaphorique de la dyscrasie des humeurs, et plus spécifiquement de la mélancolie, qui naît d'une combustion interne pour Aristote, Hippocrate, Galien ou Rufus d'Éphèse, à leur tour longuement repris ${ }^{85}$. L'échauffement de la

80 Selon le Trésor de la langue française, l'emploi figuré d'alambic — " poésie à l'alambic" - apparaît avec sa connotation péjorative au début du XIx ${ }^{\mathrm{e}}$ siècle; au même moment, pareil déplacement connotatif atteint "alambiquer", qui paraît être tombé en désuétude dans le dernier quart du XviI ${ }^{\mathrm{e}}$ siècle.

81 Je reprends le concept à Dominique Maingueneau, op. cit., p. 28.

82 Position réactionnaire qu'est venu démentir le livre de Philippe Moret, Tradition et modernité de l'aphorisme: Cioran, Reverdy, Scutenaire, Jourdan, Chazal, Genève, Librairie Droz, coll. "Histoire des Idées et Critique Littéraire", 1997.

83 Maximes et portraits, op. cit., p. 143.

84 La sentence est aussi une chimie des fleurs, comme veut la lire Claudie Balavoine, "Bouquets de fleurs et colliers de perles. Sur les recueils de formes brèves au XvI ${ }^{\mathrm{e}}$ siècle", dans Jean Lafond (dir.), op. cit., p. 51-71.

85 Voir, entre autres, Jackie Pigeaud, Folie et cures de la folie chez les médecins de l'Antiquité gréco-romaine. La manie, Paris, Société d'Édition "Les Belles Lettres", coll. Etudes Anciennes", 1987. 
mélancolie par les vapeurs du sang fait des atrabilaires et des furieux, si le sang ne vient humidifier, en surcroît, l'humeur aduste pour faire alors les hommes ingénieux ${ }^{86}$. Dans la pathologie de l'humeur mélancolique, le sang agit, selon la maxime $251 \mathrm{du}$ manuscrit Liancourt, de la même façon que l'eau qui, dans la chimie de l'alambic, réfrigère les vapeurs pour en faire une liqueur, selon la description de l'Encyclopédie.

Patrick Dandrey a très justement invité à parcourir la rhétorique quasi vierge des analogies entre la pathologie du corps et la maladie de l'âme, en précisant le rôle premier qui y tenait le "discours mélancologue "87. Par la maxime, La Rochefoucauld viserait, dans un même geste, une cure de lui-même et une thérapie universelle qui passerait par les miasmes du remède dans le mal. Le poison - est-ce la poudre de vipère dont le duc demande à Madame de Sablé de se rappeler le bon usage avant de lire des maximes nouvelles qu'il lui adresse ${ }^{88}$ ? — ou la thérapie du mal par le mal, qui est celle d'Hamlet et que préconise SainteBeuve $^{89}$ lorsqu'il définit la lecture que commandent les Maximes, assignent à la sentence sa fonction évidente de catharsis par sa brièveté qui contraste avec la longueur insensibilisante du traité, comme la tragédie vivifie par sa représentation les passions que ne peut exciter longuement le récit, ainsi que le croyait Aristote dans sa Poétique.

Nous discuterons à la première vue s'il est vrai ou non que les vices entrent souvent dans la composition de quelques vertus, comme les poisons entrent dans la composition des plus grands remèdes de la médecine. ${ }^{90}$

La maxime - ici, M 182 que je souligne - devient l'occasion du dialogue, comme si la conversation naissait, contre la violence asociale de l'CEdipe, de la dialectique - enfin recouvrée! — du mal par le mal, la seule possible dans le vivre mélancolique.

86 André Du Laurens, Les oeuvres de $M^{e}$ Du Laurens, [...]. Traduites de latin en françois par $M^{e}$ Théophile Gelée, [...], Paris, 1642, p. 294-295.

87 Patrick Dandrey, "La rédemption par les lettres", loc. cit., p. 83.

88 Euvres complètes, op. cit., p. 620.

89 Portraits de femmes, op. cit., p. 1242.

90 Euvres complètes, op. cit., p. 630-631. Lettre au Père Thomas Esprit en date du 6 février [1665] - Jean Lafond la place en 1664. Voir encore, sans exhaustivité aucune, la maxime non retenue que, parmi plusieurs autres, La Rochefoucauld livre à la méditation de Madame de Sablé: "L'extrême ennui sert à nous désennuyer" (ibid., p. 642). 
Au terme de cette lecture, la discontinuité des Maximes atteste moins une perception éclatée du monde - dont les réseaux étroits de la sociabilité sont comme tranchés par le scalpel qui y cherche des motivations égoïstes toutes contraires à l'altruiste civilité 91 - qu'un corps brisé qui se recompose en myope de lui-même. La magie d'Isis n'opère plus sous le ciel d'CEdipe. L'éclatement du texte inscrit la blessure comme traumatisme fondateur dans l'espace de la compensation, espace de langage de soi à soi. C'est ainsi que les Maximes poursuivent le projet autobiographique comme représentation de soi - et certainement pas comme imitation de soi, fadaise pour les naifs - et elles ponctuent par une solution inédite, déroutante mais enfin totalement satisfaisante les Mémoires, où La Rochefoucauld, second en tout, déporte le je autobiographique loin de la paratopie du discours sur soi qui devient un discours sur l'Autre. Les Maximes continuent l'autoportrait et, sans cesse reprises, elles distillent, par leur concision, les instants longs d'une existence qui s'exsude en une suite monadologique où le moi est soustrait au temps et aux lieux de la souffrance transfigurée sur une scène des idéaux que n'atteignent plus ni l'histoire ni l'espace humains. C'est aussi pourquoi les Maximes, qui sont nées de la mélancolie et qui sont un remède du mal par le mal, tendent à fixer dans le ciel imaginaire le soleil et à dépouiller une dernière fois Saturne de ses attributs qui, en substitution aux parties perdues, signalent les ravages du temps. Et malgré la négation la plus intime, le texte n'en finit pas de s'affirmer au partir du corps, comme instance génératrice, autre nom de la matrice ou du vaisseau au sens vieilli de l'anatomie —, de toute ouvre de pénible vie.

91 C'est la belle lecture de Louis van Delf, "Du théâtre du monde à l'anatomie", loc. cit., p. 61. 